\title{
The Design of Disturbances Rejection Controller for Decoupling Internal Model Control System of Non-Square Processes
}

\author{
Guanfei Shan ${ }^{\mathrm{a}}$, \\ Automation Institute, Beijing University of Chemical \\ Technology, Beijing 100029, China. \\ aemail: shangf412@hotmail.com,
}

\begin{abstract}
Based on the two-degree-of-freedom internal model control structure, the design of external input/output disturbances rejection controller for non-square processes is proposed in this paper. The method design different disturbance controllers by choosing different forms of external input/output disturbance, such as step disturbance, dynamic disturbance. Especially when it comes to input disturbance controller design, the design of controller needs to offset the effect of slow dynamic poles by adding constraints. Simulation results have proved the effectiveness and reliability of the proposed method.
\end{abstract}

Keywords-internal model control (IMC), non-square processes, disturbances rejection, two-degree-of-freedom control, relative normalized gain array (RNGA)

\section{INTRODUCTION}

In actual chemical production process, it is always need to use multiple controlled variables and multiple manipulated variables to describe a controlled system. And processes with unequal number of inputs and outputs often arise, namely it is a non-square system ${ }^{[1]}$. Meanwhile, the external disturbances is always present in actual process, so in order to get better response, it is necessary to reject the impact of these external disturbances ${ }^{[2]}$.

Internal model control ${ }^{[3]}$ is an advanced control method which can effectively solve the decoupling problems of multivariable system, however, for basic internal model control structure, the controller design is only a trade-off between tracking performance and disturbance performance. For this reason, two degree of freedom control structure ${ }^{[4]}$ is proposed which can make the set-point tracking and disturbance rejection controlled respectively.

This paper selected non-square multi-variable system as controlled object, used two degree of freedom internal model control structure, and designed the external input/output disturbances rejection controller. Then design disturbance controllers according different forms of external input/output disturbance, such as step disturbance, dynamic disturbance. Using Simulink for simulation, the results show that the method has better effect for disturbances rejection.

\section{The Structure of DeCoupling InTERnAL MODEL CONTROL}

A two-degree-of-freedom IMC control structure is

\author{
Qi Wang ${ }^{\bowtie, b}$ \\ Automation Institute, Beijing University of Chemical \\ Technology, Beijing 100029, China. \\ bemail: jinqb@mail.buct.edu.cn
}

shown in Fig.1.

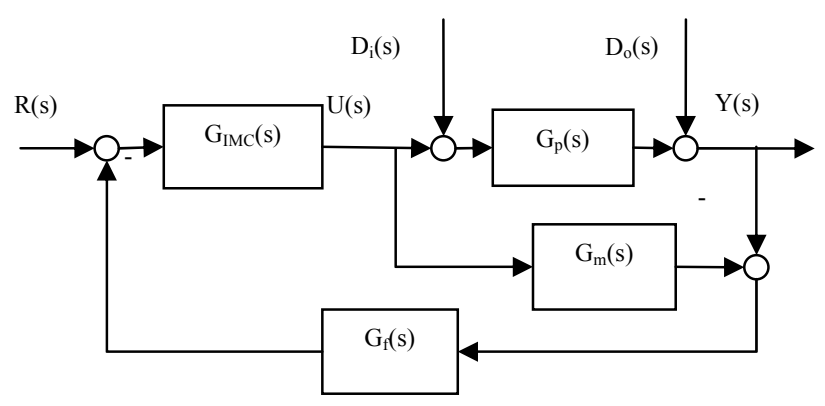

Fig.1. Two-DEGREe-OF-FrEEDOM IMC STRUCTURE

Where $G_{p}(s)$ and $G_{m}(s)$ represent the transfer functions of non-square process and the process model respectively with ' $n$ ' inputs and ' $m$ ' output $(m \leq n), G_{I M C}(s)$ is decoupling internal model controller with ' $m$ ' inputs and ' $n$ ' outputs. $G_{f}(s)$ is the feedback controller to realize the disturbances rejection. $Y(s), \mathrm{U}(\mathrm{s})$ and $R(s)$ are the process output, input and set-point respectively. $D_{i}(s)$ and $D_{o}(s)$ are the external input disturbance and external output disturbance respectively.

Based on Fig.1, the transfer function matrix $H(s)$ between set-point $R(s)$ and output $Y(s)$ can be represented as

$$
H(s)=G_{p}(s)\left[I+G_{I M C}(s)\left(G_{p}(s)-G_{m}(s)\right)\right]^{-1} G_{I M C}(s)
$$

If the model describes the plant exactly, namely $G_{p}(s)=G_{m}(s)$, then $H(s)$ becomes:

$$
H(s)=G_{p}(s) G_{I M C}(s)=G_{m}(s) G_{I M C}(s)
$$

Then the system is decoupled if and only if $H(s)$ is decoupled (diagonal and non-singular), and according to the method described in literature [5], internal model controller can be design as follows: 
$\mathrm{H}(\mathrm{s})=G_{m+}(s) F(s)=\operatorname{diag}\left\{h_{i i}\right\} ; h_{i i} \neq 0 ; \mathrm{i}=1,2,3 \cdots \mathrm{m}$

where the low pass filter is $\mathrm{F}=\operatorname{diag}\left\{\frac{1}{\left(\lambda_{i} s+1\right)^{n_{i}}}, i=1,2, \cdots, m\right\}$, and process model non-minimum phase can be described as $G_{m+}=\operatorname{diag}\left\{e^{-\theta_{K i} s} \prod_{1}^{M_{i}}\left(\frac{-\mathrm{s}+z_{p}}{s+z_{p}^{*}}\right)^{W_{i}}, \mathrm{i}=1,2, \cdots, \mathrm{m}\right\}$, where $\theta_{K i}$ is the maximum predictive term in the $i$-th column of pseudo-inverse $G_{m-}^{+1}$ and $Z_{p}$ is the right half-plane poles in corresponding column, and $Z_{p}^{*}$ is its conjugate complex, and $W_{i}$ is the number of the same pole, while $M_{i}$ is the number of different poles exist in the $i$-th column of $G_{m-}^{+1}$.

The transfer function matrix between

$D_{o}(s)$ and $Y(s)$ can be written as

$$
H_{d o}=\left[I-G_{p}(s)\left[-G_{I M C}(s) G_{f}(s)\left(I-G_{m}(s) G_{I M C}(s) G_{f}(s)\right)^{-1}\right]\right]^{-1}
$$

The transfer function matrix between $D_{i}(s)$ and output $Y(s)$ can be expressed as:

$$
H_{d i}=\left[I-G_{p}(s)\left[-G_{I M C}(s) G_{f}(s)\left(I-G_{m}(s) G_{I M C}(s) G_{f}(s)\right)^{-1}\right]\right]^{-1} G_{p}(s)
$$

It can be seen that the disturbance rejection performance is decided by $G_{I M C}(s)$ and $G_{f}(s)$, while $G_{I M C}(s)$ can be designed to realize the set-point tracking, so it just need to design $G_{f}(s)$ to reject disturbance.

\section{THE DESIGN OF DISTURBANCES REJECTION CONTROLLER}

\section{A. Output disturbances rejection controller}

The transfer function matrix between

$D_{o}(s)$ and $Y(s)$ can be written as:

$$
\begin{aligned}
& H_{d o}(s)=\left[I-G_{p}(s)\left[-G_{I M C}(s) G_{f}(s)\left(I-G_{m}(s) G_{I M C}(s) G_{f}(s)\right)^{-1}\right]\right]^{-1} \\
& =\left[I-\left[-H(s) G_{f}(s)\left(I-H(s) G_{f}(s)\right)^{-1}\right]\right]^{-1}
\end{aligned}
$$

From (6), it can be seen that other elements are diagonal except $G_{f}(s)$, so we design $G_{f}(s)$ which is also diagonal. Then (6) can be further simplified as follows:

$$
H_{d o}(s)=I-H(s) G_{f}(s)=I-G_{m+}(s) F(s) G_{f}(s)
$$

Considering the output disturbance response, it can be written as:

$$
Y_{d o}(s)=D_{O}(s)-G_{m+}(s) F(s) G_{f}(s) D_{O}(s)
$$

In formula (8), the first item cannot be changed, while the second item $G_{m+}(s)$ can be approximated by Pade approximate method.

$$
\begin{aligned}
& G_{m+}=\operatorname{diag}\left\{e^{-\theta_{K i} s} \prod_{1}^{D_{i}}\left(e^{-2 Z_{p} s}\right)^{W_{i}}, \mathrm{i}=1,2, \cdots, \mathrm{m}\right\} \\
& =\operatorname{diag}\left\{e^{-\varphi_{i} s} \mathrm{i}=1,2, \cdots, \mathrm{m}\right\}
\end{aligned}
$$

Thus, we can design the second item to make its response become $D_{\mathrm{O}}(s)$ with delaying $\varphi_{i}$ time, that is to say the output disturbance response is zero after $\varphi_{i}$ time. Each output can achieve the response as following:

$$
y_{d o j}(t)=\left\{\begin{array}{l}
d_{o j}(t), t<\varphi_{j} \\
0, t \geq \varphi_{j}
\end{array}\right.
$$

Then the optimal disturbances rejection controller meets the following formula:

$$
G_{m+}(s) F(s) G_{f}(s) D_{O}(s)=\hat{D}_{O}(s)
$$

Where $\hat{D}_{\mathrm{O}}(s)$ can be expressed in time domain as follows:

$$
\begin{aligned}
& \hat{D}_{O}(t)=\operatorname{diag}\left\{\hat{d}_{o 1}(t), \cdots, \hat{d}_{o j}(t), \cdots, \hat{d}_{o m}(t)\right\} \\
& =\operatorname{diag}\left\{d_{o 1}(t) \cdot 1\left(t-\varphi_{1}\right), \cdots, d_{o m}(t) \cdot 1\left(t-\varphi_{m}\right)\right\} \\
& =D_{O}(t) \operatorname{diag}\left\{1\left(t-\varphi_{1}\right), \cdots, 1\left(t-\varphi_{j}\right), \cdots, 1\left(t-\varphi_{m}\right)\right\}
\end{aligned}
$$

After Laplace transform, (12) will become $\hat{D}_{o}(s)$, then the optimal disturbance rejection controller can be calculated by (11).

Following, towards step disturbance and dynamic disturbance, disturbances rejection controller is designed respectively.

(1) Considering the output disturbance for the step form

$$
D_{O}(s)=\operatorname{diag}\left\{\frac{a_{o 1}}{s}, \cdots, \frac{a_{o m}}{s}\right\}
$$

After Laplace inverse transformation, the time-domain expression of $(13)$ is $D_{O}(t)=\operatorname{diag}\left\{a_{o 1}, \cdots, a_{o m}\right\}$, then take it into (12) and deduce the following formula:

$$
\begin{aligned}
& \hat{D}_{O}(t)=\operatorname{diag}\left\{a_{o 1} \cdot 1\left(t-\varphi_{1}\right), \cdots, a_{o j} \cdot 1\left(t-\varphi_{j}\right)\right. \\
& \left., \cdots, a_{o m} \cdot 1\left(t-\varphi_{m}\right)\right\}
\end{aligned}
$$

After Laplace transform, (14) becomes

$$
\begin{aligned}
& \hat{D}_{O}(s)=\operatorname{diag}\left\{\frac{a_{o 1}}{s} e^{-\varphi_{1} s}, \cdots, \frac{a_{o j}}{s} e^{-\varphi_{j} s}, \cdots, \frac{a_{o m}}{s} e^{-\varphi_{m} s}\right\} \\
& =D_{O}(s) \cdot G_{m+}(s)
\end{aligned}
$$

Take (15) into (11) and get the optimal controller

$$
\hat{G}_{f}(s)=F^{-1}(s)
$$

But the expression (16) is not regular, so filter is added. Finally the disturbances rejection controller is designed as follows:

$$
G_{f}(s)=F^{-1}(s) F_{o d}(s)
$$

(2) Considering the output disturbance for the dynamic form 


$$
D_{O}(s)=\operatorname{diag}\left\{\sum_{i} \frac{q_{i 1}}{s+p_{i 1}}, \cdots, \sum_{i} \frac{q_{i m}}{s+p_{i m}}\right\}
$$

After Laplace inverse transformation, becomes $D_{o}(t)=\operatorname{diag}\left\{\sum q_{i 1} e^{-p_{i 1} t}, \cdots, \sum q_{i m} e^{-p_{m i} t}\right\}$, then take it into (12) and get the formula as follows:

$$
\begin{aligned}
& \hat{D}_{O}(t)=\operatorname{diag}\left\{d_{o 1}\left(t-\varphi_{1}\right) e^{-p_{i 1} \varphi_{1}} \cdot 1\left(t-\varphi_{1}\right)\right. \\
& \left., \cdots, d_{o m}\left(t-\varphi_{m}\right) e^{-p_{i m} \varphi_{m}} \cdot 1\left(t-\varphi_{m}\right)\right\}
\end{aligned}
$$

After Laplace transform, (19) becomes

$$
\begin{aligned}
& \hat{D}_{O}(s)=\operatorname{diag}\left\{\sum_{i} \frac{q_{i 1} e^{-p_{i l} \varphi_{1}}}{s+p_{i 1}} e^{-\varphi_{i} s}, \cdots, \sum_{i} \frac{q_{i m} e^{-p_{i m} \varphi_{m}}}{s+p_{i m}} e^{-\varphi_{m} s}\right\} \\
& =\tilde{D}_{O}(s) \llbracket G_{m+}(s)
\end{aligned}
$$

Take (20) into (11) and get the optimal controller

$$
\hat{G}_{f}(s)=F^{-1}(s) \tilde{D}_{O}(s) D_{O}^{-1}(s)
$$

Also if (21) is not regular, add filter to correct. Then the disturbances rejection controller is designed as follows:

$$
G_{f}(s)=F^{-1}(s) \tilde{D}_{O}(s) D^{-1}(s) F_{o d}(s)
$$

In order to reject the output disturbance, the design of $G_{f}(s)$ must satisfy the following conditions:

$$
\lim _{s \rightarrow 0} s\left[I-G_{m+}(s) F(s) G_{f}(s)\right] D_{o}(s)=0
$$

B. Input disturbances rejection controller

The transfer function matrix between $D_{i}(s)$ and $Y(s)$ can be simplified as:

$$
\begin{aligned}
& H_{d i}(s)=\left[I-H(s) G_{f}(s)\right] G_{p}(s) \\
& =\left[I-G_{m+}(s) F(s) G_{f}(s)\right] G_{p}(s)
\end{aligned}
$$

Compared with the output disturbance transfer function, input disturbance transfer function has an additional multiplier that is controlled process. And if a pole of the transfer function near to the original point, it will lead to slow dynamic response. So $F_{o d}(s)$ needs to be redesigned to offset its effects. To solve this problem, the following constraint is added.

$$
\lim _{s \rightarrow p_{0}} \frac{d^{q-1}\left[I-G_{m+}(s) F(s) G_{f}(s)\right]}{d s^{q-1}}=0
$$

Where $p_{0}$ is slow dynamic pole, $q$ is the number of the same pole. Here slow dynamic pole is defined as: $\left|p_{o}\right| \leq 0.1$ and $\left|p_{o}\right| \geq 5\left|p_{k}\right|, \quad p_{k}$ is other pole.

So disturbances rejection controller $G_{f}(s)$ is redesigned as following forms:

$$
F_{\text {od }}(s)=\operatorname{diag}\left\{\frac{\sum_{d 1=0}^{q_{1}} \eta_{d 1} s^{d 1}}{\left(\lambda_{f 1} s+1\right)^{n_{1}+q_{1}}}, \cdots, \frac{\sum_{d m=0}^{q_{m}} \eta_{d m} s^{d m}}{\left(\lambda_{f m} s+1\right)^{n_{m}+q_{m}}}\right\}
$$

But $F_{o d}(s)$ is $m$-dimension diagonal matrix, while $D_{i}(s)$ is $n$-dimension vector. For non-square system $(m \neq n)$, the redesign of $F_{\text {od }}(s)$ is unable to realize. So this paper pairs the loops using relative normalized gain matrix method ${ }^{[6]}$, and selects external input disturbances with the biggest influence on system output, then design input disturbance rejection controller.

Assuming that loop pairings is selected as $1 / 1, \ldots, \mathrm{m} / \mathrm{m}$, and external input disturbances are selected as $D_{i}^{\prime}=\operatorname{diag}\left\{d_{i 1}, d_{i 2}, \cdots, d_{i m}\right\}$, then (24) can be reduced to:

$$
H_{d i}(s)=\left[I-G_{m+}(s) F(s) G_{f}(s)\right] G_{p}^{\prime}(s)
$$

Where $G_{p}^{\prime}(s)=\operatorname{diag}\left\{\mathrm{g}_{11}(\mathrm{~s}), \cdots, \mathrm{g}_{m m}(\mathrm{~s})\right\}$. At the same time, for input disturbance, after loop pairing, the design of $G_{f}(s)$ still need satisfy the following conditions:

$$
\lim _{s \rightarrow 0} s\left[I-G_{m+}(s) F(s) G_{f}(s)\right] D_{i}^{\prime}(s)=0
$$

Finally, disturbances rejection controller is designed after loop pairing, and the numerator of all diagonal elements of (26) is constant, which is determined by both (25) and (28).

\section{Simulation Results}

Example Consider the shell control problem with 3 inputs and 2 outputs ${ }^{[7]}$. The transfer function matrix is given as

$$
G_{p}(s)=\left[\begin{array}{ccc}
\frac{4.05 e^{-81 s}}{50 s+1} & \frac{1.77 e^{-84 s}}{60 s+1} & \frac{5.88 e^{-81 s}}{50 s+1} \\
\frac{5.39 e^{-54 s}}{50 s+1} & \frac{5.72 e^{-42 s}}{60 s+1} & \frac{6.9 e^{-45 s}}{40 s+1}
\end{array}\right]
$$

First of all, according to the equivalent transfer function method $^{[8]}$, the decoupling internal model controller is designed as

$$
G_{I M C}(\mathrm{~s})=\left[\begin{array}{cc}
\frac{104.0130 s+1}{12.6459(75 s+1)} e^{-3 s} & \frac{933.1931 s+1}{-316.9046(35 s+1)} \\
\frac{63.0397 s+1}{-2.9766(75 s+1)} e^{-3 s} & \frac{61.6007 s+1}{3.6356(35 s+1)} \\
\frac{37.6080 s+1}{4.6140(75 s+1)} & \frac{21.4159 s+1}{-12.4033(35 s+1)} e^{-3 s}
\end{array}\right]
$$

Non-minimum phase is

$$
G_{m+}(s)=\operatorname{diag}\left\{e^{-84 s}, e^{-45 s}\right\}
$$

Filter is selected as:

$$
\mathrm{F}=\operatorname{diag}\left\{\frac{1}{75 s+1}, \frac{1}{35 s+1}\right\}
$$

The non-square normalized relative gain matrix is

$$
\Lambda^{N}=\left[\begin{array}{ccc}
0.3203 & -0.5946 & 1.2744 \\
-0.0170 & 1.5733 & -0.5563
\end{array}\right]
$$

Then loop pairing is selected as $3 / 1,2 / 2$, and the transfer function is selected as 


$$
\tilde{G}_{p}(s)=\operatorname{diag}\left\{\frac{5.88 e^{-81 s}}{50 s+1}, \frac{5.72 e^{-42 s}}{60 s+1}\right\}
$$

Second, disturbances rejection controllers are designed respectively.

(1) Input disturbance is the unit step, that is

$$
D_{i}=\operatorname{diag}\left\{\frac{1}{\mathrm{~s}}, \frac{1}{\mathrm{~s}}, \frac{1}{\mathrm{~s}}\right\}
$$

According to loop pairing, input disturbance is selected as $D_{i}^{\prime}=\operatorname{diag}\left\{\frac{1}{S}, \frac{1}{s}\right\}$,

According (17), disturbance reject controller is designed as follows:

$$
G_{f}(s)=F^{-1}(s) F_{o d}(s)
$$

But two loops both exist slow dynamic poles $-\frac{1}{50}$ and $-\frac{1}{60}$ respectively, then $F_{\text {od }}(s)$ is designed as:

$$
\begin{aligned}
& F_{o d}(s)=\operatorname{diag}\left\{\frac{\eta_{11} s+1}{\left(\lambda_{f 1} s+1\right)^{2}}, \frac{\eta_{22} s+1}{\left(\lambda_{f 2} s+1\right)^{2}}\right\} \\
& \text { Where } \eta_{11}=(-50)\left[\left(\frac{\lambda_{f 1}}{-50}\right)^{2} \mathrm{e}^{\frac{1}{-50}}-1\right], \quad \eta_{22}=(-60)\left[\left(\frac{\lambda_{f 1}}{-60}\right)^{2} \mathrm{e}^{\frac{1}{-60}-1}\right.
\end{aligned}
$$

At a perfect matching of a process plant and model, when set-point input is zero, the input disturbance responses of $y_{1}$ and $y_{2}$ are shown in Fig.2 and Fig.3, where the solid line represents the method in this paper, the dashed line represents the method proposed in literature [5]. It can be seen from the simulation results that the method proposed in this paper shows better disturbance rejecting performance compared with the one that proposed in [5].

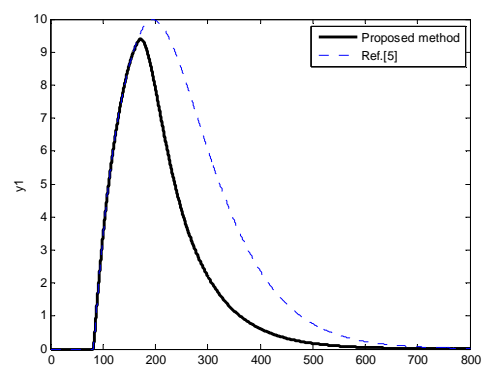

FIG.2 RESPONSES Y 1 FOR UNIT STEP INPUT DISTURBANCES

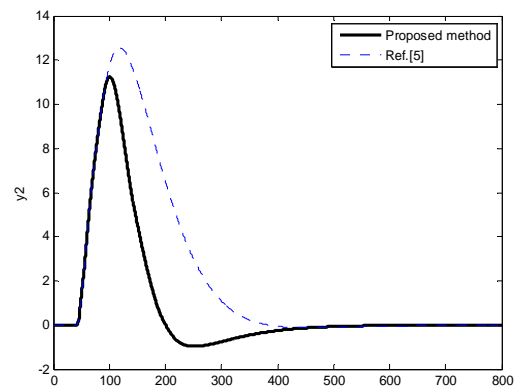

FIG.3 RESPONSES Y2 FOR UNIT STEP INPUT DISTURBANCES

While in order to verify the robustness of the proposed method, we increase the gains and the dead times of the process elements by $20 \%$, contrarily, the time constants are decreased by $20 \%$,the input disturbances rejection response is shown in Fig.4. The result shows two outputs still drive to zero, and have strong robustness in the case of the given perturbation.

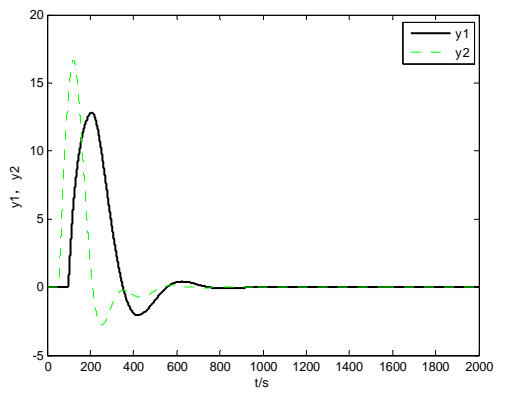

FIG.4 RESPONSES FOR UNIT STEP INPUT DisTURBANCES FOR MISMATHCH

(2) Output disturbance is dynamic form

$$
D_{o}=\operatorname{diag}\left\{\frac{1}{10 s+1}, \frac{-1}{10 s+1}\right\}
$$

According (20), it can be obtained that

$$
\tilde{D}_{O}=\operatorname{diag}\left\{\frac{e^{-\frac{84}{10}}}{10 s+1}, \frac{-e^{-\frac{45}{10}}}{10 s+1}\right\}
$$

According (22) and (23), disturbance reject controller is designed as follows:

$$
G_{f}(s)=F^{-1}(s) \tilde{D}_{o}(s) D^{-1}{ }_{o}(s) F_{o d}(s)=\operatorname{diag}\left\{\frac{75 s+1}{\lambda_{f 1} s+1}, \frac{45 s+1)}{\lambda_{f 2} s+1}\right\}
$$

At a perfect matching, and set-point input is zero, the input disturbance responses of $y_{1}$ and $y_{2}$ are shown in Fig.5 and Fig.6, while with the given perturbation, the input disturbances rejection response is shown in Fig.7. 


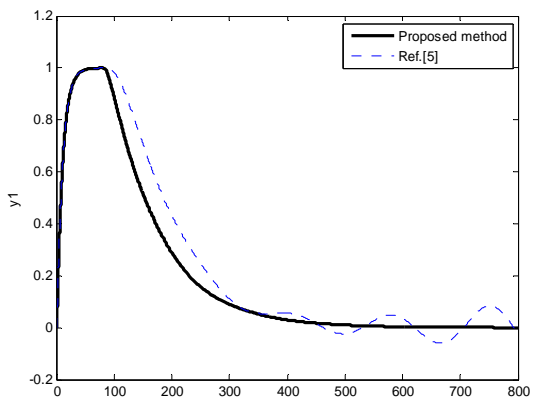

Fig.5 RESPONSES Y 1 FOR OUTPUT DISTURBANCES DO

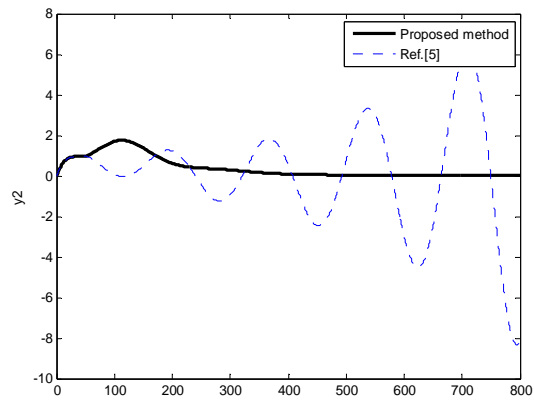

FIG.6 RESPONSES Y 2 FOR OUTPUT DISTURBANCES DO

The simulation results showed that the method proposed in this paper shows better disturbance rejecting performance and strong robustness in the case of the given perturbation compared with the one that proposed in [5].

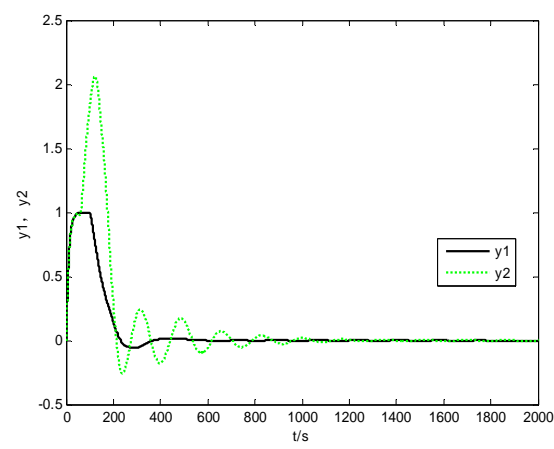

FIG.7 OUTPUT RESPONSES FOR OUTPUT DISTURBANCES DO FOR MISMATHCH

\section{V.CONCLUSION}

This paper proposed the design method of input/output disturbance rejection controller for non-square multivariable time-delay systems.

Under two-degree-of-freedom internal model control structure, it can realize the set-point tracking and system disturbances rejection control respectively. First to design decoupling internal model controller, making input and output decoupling. Then according to the transfer function between disturbance and output, different disturbance rejecting controller is designed corresponding to different disturbance form. Especially, for input disturbance, it is also need to do loop pairings and consider the effect of slow dynamic poles. Finally, using simulation results verified the effectiveness of the method.

\section{ACKNOWLEDGMENTS}

This work is partially supported by the National High-Tech Research and Development Plan of China (Grant2008AA042131), the National Grand Fundamental Research 973 Program of China (Grant 2007CB714300) and the National Natural Science Foundation of China (61273132)

\section{REFERENCES}

1] P.Y. Chen, L.L. Ou, J. Sun and W.D. Zhang: Modified internal model control and its application in non-square processes. Control and Decision vol. 23(2008), p. 581-584.

[2] Y.Y. Liu: Quantitative Analysis and Design of The Decoupling Control Systems for Multivariable Processes with Multiple Time Delays, Shanghai Jiao Tong University, Shanghai (2008).

[3] C.E. Garcia, M. Morari: Internal Model Control. 3. Multivariable control law computation and tuning guidelines. Ind. Eng. Chem. Process Des. vol.24(1985), p. 484-494

[4] L. Tao, F.R. Gao: Enhanced IMC design of load disturbance rejection for integrating and unstable processes with slow dynamics. ISA Transactions, vol. 50 (2011), p. 239-248.

[5] Q.B. Jin, Y. Guo, Z.Y. Liu, A.A. Song: Decoupling Internal Model Control for Non-square Process with Time Delays, International Conference on Measuring Technology and Mechatronics Automation (2010).

[6] M.J. He, W.J. Cai, W. Ni and L.H. Xie: RNGA based control system configuration for multivariable processes. Journal of Process Control, vol.19 (2009), p. 1036-1042.

[7] B.A. Ogunnaike, W. H. Ray: Multivariable controller design for linear systems having multiple time delays, AIChE Journal, vol.25(1979), p. 1043-1057.

[8] Q. Xiong, W.J. Cai, M.J. He: Equivalent transfer function method for PI/PID controller design of MIMO processes. Journal of Process Control, vol.17(2007), p. 665-673. 